\title{
Extension of Process Damping to Milling with Low Radial Immersion
}

\author{
Tamás G. Molnár • Tamás Insperger • Dániel Bachrathy • Gábor Stépán
}

Received: date / Accepted: date

\begin{abstract}
This paper investigates the stabilizing effect of process damping at low cutting speeds for regenerative machine tool vibrations of milling processes. The process damping is induced by a velocity-dependent cutting force model, which takes into account that the actual cutting velocity is different from the nominal one during machine tool vibrations. The chip thickness and the cutting force are calculated according to the direction of the actual cutting velocity. This results in an additional damping term in the governing delaydifferential equation, which is time-periodic for milling and inversely proportional to the cutting speed. In the literature, this term is often assumed to be constant and is considered to improve stability properties at low spindle speeds. In this paper, it is shown that the velocitydependent cutting force model captures the improvement in the low-speed stability only for turning operations and milling with large radial immersion, while it results in a negative process damping term for low-
\end{abstract}

This work has been supported by the ÚNKP-16-3-I. New National Excellence Program of the Ministry of Human Capacities. This work was supported by the Hungarian National Science Foundation under grant OTKA-K105433. The research leading to these results has received funding from the European Research Council under the European Union's Seventh Framework Programme (FP/2007-2013) / ERC Advanced Grant Agreement n. 340889.

Tamás G. Molnár, Dániel Bachrathy, Gábor Stépán

Department of Applied Mechanics, Budapest University of Technology and Economics, H-1111 Budapest, Hungary

E-mail: molnar@mm.bme.hu, bachrathy@mm.bme.hu, stepan@mm.bme.hu

Tamás Insperger

Department of Applied Mechanics, Budapest University of Technology and Economics and MTA-BME Lendület Human Balancing Research Group, H-1111 Budapest, Hungary

E-mail: insperger@mm.bme.hu immersion milling. Consequently, an extended process damping model is needed to explain the low-speed stability improvement for low radial immersion milling.

Keywords Metal cutting · Milling - Machine tool chatter $\cdot$ Cutting force $\cdot$ Process damping

\section{Introduction}

Improving the productivity and the accuracy of metal cutting operations is of high importance in manufacturing technology. One important barrier of increasing the achievable material removal rate and machined surface quality is the occurrence of harmful vibrations known as machine tool chatter. Modeling the dynamics of machine tool vibrations is therefore an active field of research.

The first models that succeeded in describing the onset of chatter appeared in the 1950's when Tobias [1] and Tlusty [2] introduced the theory of regenerative machine tool vibrations. This concept uses delaydifferential equations to describe the regeneration of the waviness of the machined surface during the consecutive cuts. The stability analysis of the delay-differential equations gives the so-called stability lobe diagrams (or stability charts), which identify the chatter-free technological parameter regions in the plane of the spindle speed and the depth of cut. These diagrams help the machinist in selecting the desired technological parameters associated with optimal material removal rate without experiencing machine tool vibrations.

According to experimental results [3-10], the stability boundaries (or stability lobes) shift towards higher depths of cut at low spindle speeds resulting in a larger stable (or chatter-free) region. In this article, we refer to this phenomenon as low-speed stability improve- 
ment. For the experimental data verifying the low-speed stability improvement, the reader is referred to [3-10]. Most mechanical models of metal cutting dedicate the low-speed stability improvement to an additional dissipative force during cutting, which is inversely proportional to the spindle speed. This additional term is often called as process damping [3-15]. The process damping is often explained by the contact between the tool's flank face and the wavy surface of the workpiece [3$6,8-12,15]$ or by introducing velocity-dependent cutting force models $[4,14]$. Another possible explanation for the low-speed stability improvement phenomenon is the so-called short regenerative effect $[16,17]$, which dedicates the stability improvement to the distribution of the cutting force along the rake face.

A widely accepted model to explain the origin of the process damping term is a velocity-dependent cutting force model, which is described in [4, 14] for orthogonal cutting. Namely, it is taken into account that the actual cutting velocity is different from the nominal cutting velocity during machine tool vibrations. Therefore, the thickness of the chip is calculated according to the direction of the actual cutting velocity instead of the nominal one. This results in a velocitydependent chip thickness expression, which determines the magnitude of the cutting-force components. The model also involves velocity-dependent projections of the cutting force into tangential and feed components according to the direction of the actual cutting velocity. The velocity-dependent chip thickness expression and the cutting force projections result in a process damping force in the model of cutting. In this paper, we refer to this as velocity-dependent cutting force model, and we extend this model to milling operations, especially for low radial immersion ones. The velocity-dependent chip thickness expression for milling has already been derived in [18]. Hence we use the results of [18] for the chip thickness and apply the method of $[4,14]$ to compute the components of the cutting force for milling operations.

The rest of the paper is organized as follows. As a motivation, Sect. 2 demonstrates the effect of process damping for turning operations. Section 3 introduces the single-degree-of-freedom mechanical model of milling, and gives expressions for the cutting velocity, the chip thickness, and the cutting force in the presence of machine tool vibrations. The linearized equation of motion is derived in Sect. 4, and the corresponding stability lobe diagrams are computed in Sect. 5. Section 6 gives a geometric illustration of the direction of the arising process damping force. Finally, conclusions are drawn in Sect. 7.

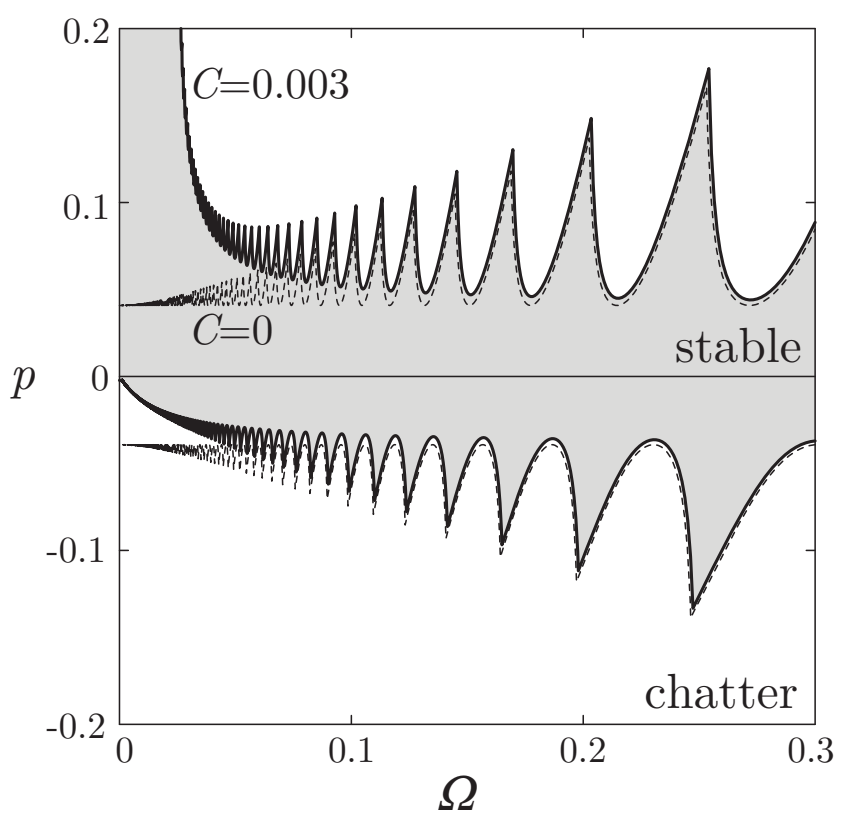

Fig. 1 Stability lobe diagram of turning operations with (solid line) and without (dashed line) process damping

\section{Motivation}

The most common way of modeling the low-speed stability improvement phenomenon is adding a damping term (the so-called process damping) to the governing equation of the cutting process, where the damping is inversely proportional to the spindle speed. This model is presented for turning operations in [14], see Eqs. (4.21)-(4.22). The corresponding dimensionless equation of tool motion can be written in the form

$\xi^{\prime \prime}(t)+2 \zeta \xi^{\prime}(t)+\xi(t)=p(\xi(t-\tau)-\xi(t))-p \tau C \xi^{\prime}(t)$,

where $p$ is the dimensionless chip width, and $\tau$ is the regenerative delay, which is inversely proportional to the dimensionless spindle speed $\Omega$ as $\tau=2 \pi / \Omega$. Parameter $\zeta$ is the damping ratio of the dominant vibration mode of the machining system, parameter $C$ is the dimensionless process damping coefficient, and the last term in Eq. (1) is the process damping term.

The stability boundaries of Eq. (1), which separate the chatter-free technological parameter regions from those with machine tool chatter, are given by [19]

$$
\begin{aligned}
\Omega(\psi) & =\frac{2 \pi \omega(\psi)}{\psi} \\
p(\psi) & =-\frac{2 \zeta \omega(\psi)}{C \psi+\sin \psi}, \\
\omega(\psi) & =-\zeta \frac{1-\cos \psi}{C \psi+\sin \psi}+\sqrt{\zeta^{2}\left(\frac{1-\cos \psi}{C \psi+\sin \psi}\right)^{2}+1}
\end{aligned}
$$




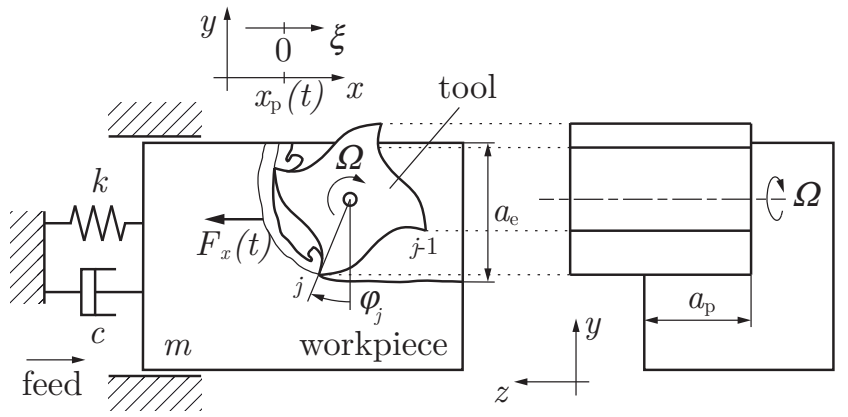

Fig. 2 Single-degree-of-freedom mechanical model of milling

Here, $\omega$ denotes the approximate angular frequency of chatter and $\omega \tau=: \psi \in[0, \infty)$ is the regenerative phase shift between the waves on the undulated machined surface created by the tool during chatter.

The corresponding stability lobe diagram is shown in Fig. 1 for $\zeta=0.02$ and $C=0.003$. The stability boundaries shift upwards at small spindle speeds in the physically meaningful half-plane $p>0$, while the stable region shrinks for $p<0$. Although negative chip width $(p<0)$ is physically meaningless in turning operations, it becomes important for milling. The stability boundaries of up-milling processes originate in the stability lobes of turning with positive chip width $(p>0)$, whereas those of down-milling come from the lobes of turning with negative chip width $(p<0)[20]$. That is, we get qualitatively the same lobes for up-milling with a large number of cutting teeth as for turning with $p>0$. Similarly, the lobes of turning in the negative half-plane $p<0$ are valid for down-milling with a large number of cutting teeth.

The phenomenon shown in Fig. 1 can be explained by the fact that the sign of the process damping term in Eq. (1) depends also on the sign of the chip width $p$. Therefore, special care must be taken to get an exact description of the process damping term when introducing it for milling operations. The physical explanation of low-speed stability improvement should be associated with a positive damping term, otherwise stability decreases at low speeds. Motivated by Fig. 1, hereinafter we investigate the sign of process damping for a milling model with velocity-dependent cutting force expression.

\section{Mechanical Model of Milling}

Consider the single-degree-of-freedom mechanical model of milling shown in Fig. 2. The motion of the workpiece relative to the cutting tool is described by the general coordinate $x$ as a function of time $t$. The motion is governed by the second-order differential equation

$m \ddot{x}(t)+c \dot{x}(t)+k x(t)=-F_{x}(t)$,

where $m, c$, and $k$ are the modal mass, damping, and stiffness parameters, respectively, corresponding to the dominant vibration mode of the machine tool-workpiece system. Here, $F_{x}$ denotes the $x$-directional component of the cutting force acting on the mill. In order to model the cutting force on the right-hand side, first we derive expressions for the velocity of the cutting edges and for the chip thickness.

\subsection{Velocity of the Cutting Edges}

Consider an $N$-fluted milling tool of radius $R$ rotating with angular velocity $\Omega$. The angular position of the $j$ th tooth of the mill is given by

$\tilde{\varphi}_{j}(t)=\Omega t+(j-1) \frac{2 \pi}{N}$

$j=1,2, \ldots, N$, see Fig. 3a for rake angle $\alpha_{\mathrm{r}}$.

In the absence of vibrations, the velocity of the $j$ th tooth tip is

$\tilde{\mathbf{v}}_{j}(t)=\left[\begin{array}{c}-R \Omega \cos \tilde{\varphi}_{j}(t) \\ R \Omega \sin \tilde{\varphi}_{j}(t) \\ 0\end{array}\right]$

provided that the feed velocity is negligible compared to the nominal cutting speed $\tilde{v}_{j}(t)=\left|\tilde{\mathbf{v}}_{j}(t)\right| \equiv R \Omega$.

From this point on, tilde indicates quantities related to the nominal cutting velocity $\tilde{\mathbf{v}}_{j}(t)$. Symbols without tilde are associated with the actual cutting velocity $\mathbf{v}_{j}(t)$ of the $j$ th tooth, where the vibration velocity $\dot{x}(t)$ of the workpiece is also taken into account, cf. Fig. 3a. The relative velocity between the tool and the workpiece therefore becomes

$\mathbf{v}_{j}(t)=\left[\begin{array}{c}-R \Omega \cos \tilde{\varphi}_{j}(t)-\dot{x}(t) \\ R \Omega \sin \tilde{\varphi}_{j}(t) \\ 0\end{array}\right]=\left[\begin{array}{c}-v_{j}(t) \cos \varphi_{j}(t) \\ v_{j}(t) \sin \varphi_{j}(t) \\ 0\end{array}\right]$

where the feed velocity is neglected compared to $R \Omega$. That is, the magnitude of the velocity changes from $\tilde{v}_{j}(t) \equiv R \Omega$ to

$$
v_{j}(t)=\sqrt{\left(R \Omega \cos \tilde{\varphi}_{j}(t)+\dot{x}(t)\right)^{2}+\left(R \Omega \sin \tilde{\varphi}_{j}(t)\right)^{2}},
$$



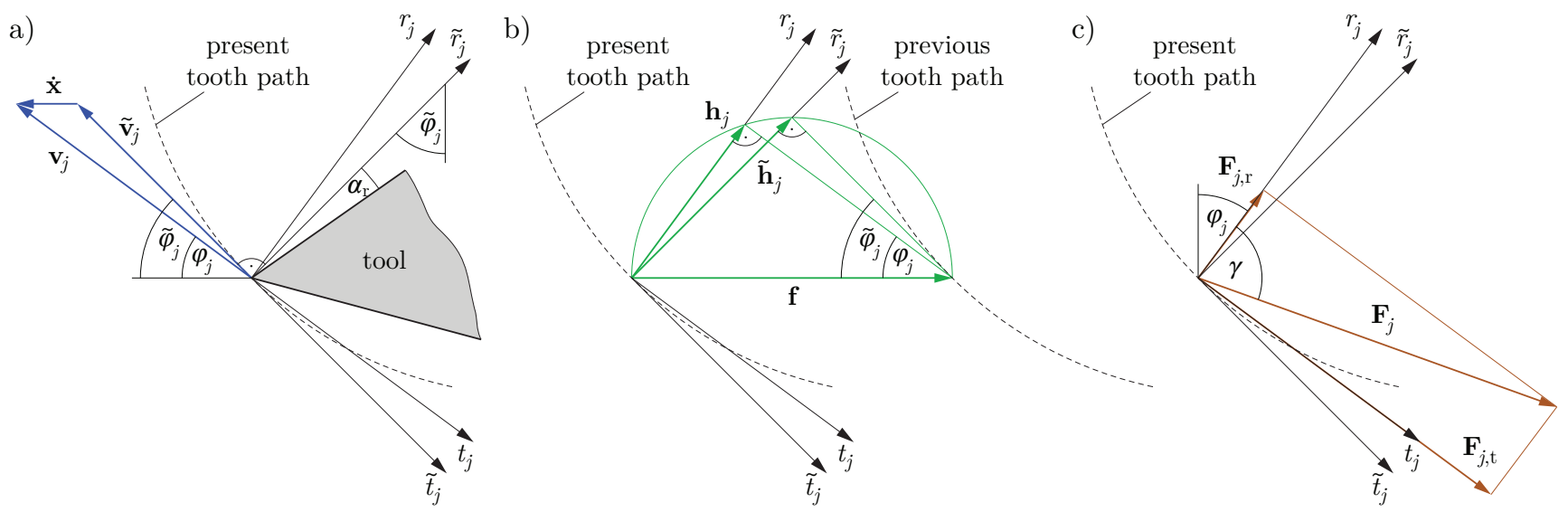

Fig. 3 Components of the velocity of the $j$ th tooth tip (a); relation of the chip thickness and the feed per tooth (b); projection of the cutting force to tangential and radial directions (c)

and its instantaneous direction is given by $\varphi_{j}(t)$ instead of the nominal $\tilde{\varphi}_{j}(t)$, satisfying

$$
\begin{aligned}
\cos \varphi_{j}(t) & =\frac{R \Omega \cos \tilde{\varphi}_{j}(t)+\dot{x}(t)}{\sqrt{\left(R \Omega \cos \tilde{\varphi}_{j}(t)+\dot{x}(t)\right)^{2}+\left(R \Omega \sin \tilde{\varphi}_{j}(t)\right)^{2}}}, \\
\sin \varphi_{j}(t) & =\frac{R \Omega \sin \tilde{\varphi}_{j}(t)}{\sqrt{\left(R \Omega \cos \tilde{\varphi}_{j}(t)+\dot{x}(t)\right)^{2}+\left(R \Omega \sin \tilde{\varphi}_{j}(t)\right)^{2}}} .
\end{aligned}
$$

A key point of this analysis is that the tangential and the radial directions are defined based on the cutting velocity. The nominal tangential and the nominal radial directions are parallel and perpendicular to the nominal cutting velocity $\tilde{\mathbf{v}}_{j}(t)$, respectively. These directions are given by the unit vectors $\tilde{\mathbf{t}}_{j}(t)$ and $\tilde{\mathbf{r}}_{j}(t)$. Similarly, in the presence of machine tool vibrations, the actual tangential and radial directions are associated with the actual cutting velocity $\mathbf{v}_{j}(t)$ and are assigned by the unit vectors $\mathbf{t}_{j}(t)$ and $\mathbf{r}_{j}(t)$. The above vectors are of form

$\tilde{\mathbf{t}}_{j}(t)=-\frac{\tilde{\mathbf{v}}_{j}(t)}{\tilde{v}_{j}(t)}=\left[\begin{array}{c}\cos \tilde{\varphi}_{j}(t) \\ -\sin \tilde{\varphi}_{j}(t) \\ 0\end{array}\right]$,

$\tilde{\mathbf{r}}_{j}(t)=\mathbf{k} \times \tilde{\mathbf{t}}_{j}(t)=\left[\begin{array}{c}\sin \tilde{\varphi}_{j}(t) \\ \cos \tilde{\varphi}_{j}(t) \\ 0\end{array}\right]$,

$\mathbf{t}_{j}(t)=-\frac{\mathbf{v}_{j}(t)}{v_{j}(t)}=\left[\begin{array}{c}\cos \varphi_{j}(t) \\ -\sin \varphi_{j}(t) \\ 0\end{array}\right]$,

$\mathbf{r}_{j}(t)=\mathbf{k} \times \mathbf{t}_{j}(t)=\left[\begin{array}{c}\sin \varphi_{j}(t) \\ \cos \varphi_{j}(t) \\ 0\end{array}\right]$

where $\mathbf{k}$ is the $z$-directional unit vector and $\times$ indicates cross product.

\subsection{Chip Thickness Expression}

Consider a milling operation with nominal feed per tooth $f_{z}$. According to the theory of regenerative machine tool vibrations, when chatter occurs, the actual feed per tooth is modified by the actual position $x(t)$ of the workpiece and its position $x(t-\tau)$ at the previous cut. That is, the actual feed per tooth is given by the feed vector

$\mathbf{f}(t)=\left[\begin{array}{c}f_{z}+x(t)-x(t-\tau) \\ 0 \\ 0\end{array}\right]$,

where $\tau$ is the regenerative delay or, equivalently, the tooth-passing period: $\tau=2 \pi /(N \Omega)$.

The chip thickness is given by the radial component of the feed vector, see Fig. 3b. If the nominal cutting velocity is used for reference as in standard models [2126 , then the chip thickness can be given as the projection of the feed vector to the nominal radial direction $\tilde{\mathbf{r}}_{j}(t)$ as $[20]$

$\tilde{h}_{j}(t)=\mathbf{f}(t) \cdot \tilde{\mathbf{r}}_{j}(t)=\left(f_{z}+x(t)-x(t-\tau)\right) \sin \tilde{\varphi}_{j}(t)$,

where - indicates scalar product. Alternatively, if the dependence of the velocity of the $j$ th tooth tip on the vibration velocity $\dot{x}(t)$ is taken into account [18], then a velocity-dependent chip thickness can be defined as

$h_{j}(t)=\mathbf{f}(t) \cdot \mathbf{r}_{j}(t)=\left(f_{z}+x(t)-x(t-\tau)\right) \sin \varphi_{j}(t)$,

in which $\varphi_{j}(t)$ depends on $\dot{x}(t)$ through Eq. (8). Note that it is a widely accepted concept [14] to define the chip thickness in the direction perpendicular to the actual cutting velocity (given by Eq. (12)) instead of 
the direction towards the center of the mill (given by Eq. (11)). Changes in the cutting velocity modify the cutting direction and hence the effective chip thickness. This concept is a possible explanation of the low-speed stability improvement for turning [14]. In this paper, we generalized this model from turning to milling.

\subsection{Projections of the Cutting Force}

Let us decompose the cutting force $\mathbf{F}_{j}(t)$ acting on the $j$ th tooth into actual tangential and radial components $\mathbf{F}_{j, \mathrm{t}}(t)$ and $\mathbf{F}_{j, \mathrm{r}}(t)$, respectively, cf. Fig. 3c. These cutting-force components are functions of the chip thickness $h_{j}(t)$ according to the cutting force characteristics. In this paper, we investigate the well-known power law characteristics, which relates the cutting force magnitude to the $q$ th power of the chip thickness:

$\mathbf{F}_{j, \mathrm{t}}(t)=g_{j}(t) K_{\mathrm{t}} a_{\mathrm{p}} h_{j}^{q}(t) \mathbf{t}_{j}(t)$,

$\mathbf{F}_{j, \mathrm{r}}(t)=g_{j}(t) K_{\mathrm{r}} a_{\mathrm{p}} h_{j}^{q}(t) \mathbf{r}_{j}(t)$,

where $K_{\mathrm{t}}$ and $K_{\mathrm{r}}$ are tangential and radial cutting-force coefficients, and $a_{\mathrm{p}}$ is the axial depth of cut. Eq. (13) yields a linear cutting force characteristics for $q=1$ and implies the widely-used three-quarter rule for $q=3 / 4$. Note that the angle between the radial cutting-force component $\mathbf{F}_{j, \mathrm{r}}(t)$ and the cutting force $\mathbf{F}_{j}(t)$ is assumed to be constant, $\gamma=\arctan \left(K_{\mathrm{t}} / K_{\mathrm{r}}\right)$, independently of time and of the vibration velocity $\dot{x}(t)$.

In Eq. (13), the coefficient $g_{j}(t)$ is a screen function, which gives 1 if the $j$ th tooth is currently engaged in cutting and 0 otherwise:

$g_{j}(t)= \begin{cases}1 & \text { if } \varphi_{\mathrm{en}}<\left(\tilde{\varphi}_{j}(t) \bmod 2 \pi\right)<\varphi_{\mathrm{ex}} \\ 0 & \text { otherwise }\end{cases}$

where mod is the modulo function, whereas $\varphi_{\text {en }}$ and $\varphi_{\text {ex }}$ denote the angular positions where the teeth enter and exit the workpiece. Given the radial immersion $a_{\mathrm{e}}$ and the tool diameter $D=2 R$, these angles can be expressed in the form

$\varphi_{\mathrm{en}}=0, \varphi_{\mathrm{ex}}=\arccos \left(1-\frac{2 a_{\mathrm{e}}}{D}\right) \quad$ for up-milling, $\varphi_{\mathrm{en}}=\arccos \left(\frac{2 a_{\mathrm{e}}}{D}-1\right), \varphi_{\mathrm{ex}}=\pi \quad$ for down-milling.

We can calculate the $x$-directional component of the cutting force from the tangential and the radial components by a scalar product with the $x$-directional unit vector i. Using Eqs. (9), (12) and (13), and summing the forces on each tooth of the mill, we obtain

$$
\begin{aligned}
F_{x}(t)= & \sum_{j=1}^{N}\left(\mathbf{F}_{j, \mathrm{t}}(t) \cdot \mathbf{i}+\mathbf{F}_{j, \mathrm{r}}(t) \cdot \mathbf{i}\right) \\
= & \sum_{j=1}^{N} g_{j}(t) a_{\mathrm{p}}\left(K_{\mathrm{t}} \cos \varphi_{j}(t)+K_{\mathrm{r}} \sin \varphi_{j}(t)\right) \\
& \quad \times \sin ^{q} \varphi_{j}(t)\left(f_{z}+x(t)-x(t-\tau)\right)^{q} .
\end{aligned}
$$

Equations (3) and (16) form a nonlinear delay-differential equation with time-periodic coefficients. Notice that the equation is nonlinear even for a linear cutting force characteristics $(q=1)$, since the angle $\varphi_{j}(t)$ used for projecting the chip thickness and the cutting-force components depends on the vibration velocity $\dot{x}(t)$ according to Eq. (8). We will analyze the effect of this velocity dependency on the linear stability properties.

\section{Linearized Equation of Motion}

We assume that Eq. (3) with (16) has a $\tau$-periodic particular solution (forced vibration) $x_{\mathrm{p}}(t)=x_{\mathrm{p}}(t+\tau)$, which is associated with the chatter-free motion. This solution satisfies

$$
\begin{aligned}
& m \ddot{x}_{\mathrm{p}}(t)+c \dot{x}_{\mathrm{p}}(t)+k x_{\mathrm{p}}(t)=-\sum_{j=1}^{N} g_{j}(t) a_{\mathrm{p}} f_{z}^{q} \\
& \quad \times\left.\left(\left.K_{\mathrm{t}} \cos \varphi_{j}(t)\right|_{\dot{x}_{\mathrm{p}}}+\left.K_{\mathrm{r}} \sin \varphi_{j}(t)\right|_{\dot{x}_{\mathrm{p}}}\right) \sin ^{q} \varphi_{j}(t)\right|_{\dot{x}_{\mathrm{p}}},
\end{aligned}
$$

where $\dot{x}_{\mathrm{p}}$ in the lower index stands for the substitution $\dot{x}(t)=\dot{x}_{\mathrm{p}}(t)$. Equation (17) is a nonlinear nonhomogeneous ODE with $\tau$-periodic coefficients.

Machine tool vibrations correspond to the loss of stability of the periodic solution $x_{\mathrm{p}}(t)$. In what follows, we analyze the stability of this periodic solution without determining $x_{\mathrm{p}}(t)$ itself, since we assume that in the parameter region under investigation $\left|\dot{x}_{\mathrm{p}}(t)\right| \ll R \Omega$ and can be neglected. For more details about the behavior of the periodic solution, the reader is referred to [18]. Now we linearize Eq. (3) with (16) around the periodic solution $x_{\mathrm{p}}(t)$. From Eq. (8), the trigonometric terms in Eq. (16) and their derivatives with respect to $x$ and 
$\dot{x}$ can be obtained in the form

$$
\begin{aligned}
& \left.\cos \varphi_{j}(t)\right|_{\dot{x}_{\mathrm{p}}} \approx \cos \tilde{\varphi}_{j}(t),\left.\quad \sin \varphi_{j}(t)\right|_{\dot{x}_{\mathrm{p}}} \approx \sin \tilde{\varphi}_{j}(t), \\
& \left.\frac{\partial \cos \varphi_{j}(t)}{\partial x}\right|_{\dot{x}_{\mathrm{p}}}=0,\left.\quad \frac{\partial \sin \varphi_{j}(t)}{\partial x}\right|_{\dot{x}_{\mathrm{p}}}=0, \\
& \left.\frac{\partial \cos \varphi_{j}(t)}{\partial \dot{x}}\right|_{\dot{x}_{\mathrm{p}}} \approx \frac{1}{R \Omega} \sin ^{2} \tilde{\varphi}_{j}(t), \\
& \left.\frac{\partial \sin \varphi_{j}(t)}{\partial \dot{x}}\right|_{\dot{x}_{\mathrm{p}}} \approx-\frac{1}{R \Omega} \sin \tilde{\varphi}_{j}(t) \cos \tilde{\varphi}_{j}(t), \\
& \left.\frac{\partial\left(\cos \varphi_{j}(t) \sin ^{q} \varphi_{j}(t)\right)}{\partial \dot{x}}\right|_{\dot{x}_{\mathrm{p}}} \approx \frac{1}{R \Omega} \sin ^{2} \tilde{\varphi}_{j}(t) \sin ^{q} \tilde{\varphi}_{j}(t) \\
& +\cos \tilde{\varphi}_{j}(t) q \sin ^{q-1} \tilde{\varphi}_{j}(t)\left(-\frac{1}{R \Omega} \sin \tilde{\varphi}_{j}(t) \cos \tilde{\varphi}_{j}(t)\right), \\
& \frac{\left.\partial \sin ^{q+1} \varphi_{j}(t)\right|_{\dot{x}_{\mathrm{p}}}}{\partial \dot{x}} \approx(q+1) \sin ^{q} \tilde{\varphi}_{j}(t) \\
& \times\left(-\frac{1}{R \Omega} \sin \tilde{\varphi}_{j}(t) \cos \tilde{\varphi}_{j}(t)\right),
\end{aligned}
$$

where the vibration velocity $\dot{x}_{\mathrm{p}}(t)$ was neglected compared to the nominal cutting speed component $R \Omega \cos \tilde{\varphi}_{j}(t)$ to simplify the analysis. From Eqs. (16) and (18), the corresponding derivatives of the cutting force become

$$
\begin{array}{r}
\left.\frac{\partial F_{x}(t)}{\partial x}\right|_{\dot{x}_{\mathrm{p}}}=\sum_{j=1}^{N} g_{j}(t) a_{\mathrm{p}}\left(K_{\mathrm{t}} \cos \tilde{\varphi}_{j}(t)+K_{\mathrm{r}} \sin \tilde{\varphi}_{j}(t)\right) \\
\times \sin ^{q} \tilde{\varphi}_{j}(t) q f_{z}^{q-1} \\
\left.\frac{\partial F_{x}(t)}{\partial \dot{x}}\right|_{\dot{x}_{\mathrm{p}}}=\sum_{j=1}^{N} g_{j}(t) a_{\mathrm{p}}\left(K_{\mathrm{t}}\left(\sin ^{2} \tilde{\varphi}_{j}(t)-q \cos ^{2} \tilde{\varphi}_{j}(t)\right)\right. \\
\left.-K_{\mathrm{r}}(1+q) \sin \tilde{\varphi}_{j}(t) \cos \tilde{\varphi}_{j}(t)\right) \sin ^{q} \tilde{\varphi}_{j}(t) \frac{f_{z}^{q}}{R \Omega}
\end{array}
$$

Considering small perturbations $\xi(t)$ around the periodic solution $x_{\mathrm{p}}(t)$, Eq. (3) with (16) can be linearized as

$$
\begin{aligned}
m \ddot{\xi}(t) & +c \dot{\xi}(t)+k \xi(t) \\
\quad= & -\left.\frac{\partial F_{x}(t)}{\partial x}\right|_{\dot{x}_{\mathrm{p}}}(\xi(t)-\xi(t-\tau))-\left.\frac{\partial F_{x}(t)}{\partial \dot{x}}\right|_{\dot{x}_{\mathrm{p}}} \dot{\xi}(t) .
\end{aligned}
$$

Using Eq. (19), and dividing by the modal mass $m$, one obtains the linear equation of motion in the form

$$
\begin{aligned}
& \ddot{\xi}(t)+2 \zeta \omega_{\mathrm{n}} \dot{\xi}(t)+\omega_{\mathrm{n}}^{2} \xi(t) \\
& =-H G_{1}(t)(\xi(t)-\xi(t-\tau))-H \frac{f_{z}}{R \Omega} G_{2}(t) \dot{\xi}(t),
\end{aligned}
$$

where $\omega_{\mathrm{n}}=\sqrt{k / m}$ is the natural angular frequency, $\zeta=c /\left(2 m \omega_{\mathrm{n}}\right)$ is the damping ratio, $H=K_{\mathrm{r}} a_{\mathrm{p}} q f_{z}^{q-1} / m$ is the specific cutting-force coefficient, and $G_{1}(t)$ and $G_{2}(t)$ are the following $\tau$-periodic coefficients:

$$
\begin{aligned}
G_{1}(t)= & \sum_{j=1}^{N} g_{j}(t)\left(\frac{K_{\mathrm{t}}}{K_{\mathrm{r}}} \cos \tilde{\varphi}_{j}(t)+\sin \tilde{\varphi}_{j}(t)\right) \sin ^{q} \tilde{\varphi}_{j}(t), \\
G_{2}(t)=\sum_{j=1}^{N} g_{j}(t) & \left(\frac{K_{\mathrm{t}}}{K_{\mathrm{r}}}\left(\frac{1}{q} \sin ^{2} \tilde{\varphi}_{j}(t)-\cos ^{2} \tilde{\varphi}_{j}(t)\right)\right. \\
& \left.-\frac{1+q}{q} \sin \tilde{\varphi}_{j}(t) \cos \tilde{\varphi}_{j}(t)\right) \sin ^{q} \tilde{\varphi}_{j}(t) .
\end{aligned}
$$

For a linear cutting force model $(q=1)$, these expressions simplify to

$$
\begin{array}{r}
G_{1}(t)=\sum_{j=1}^{N} g_{j}(t)\left(\frac{K_{\mathrm{t}}}{K_{\mathrm{r}}} \cos \tilde{\varphi}_{j}(t)+\sin \tilde{\varphi}_{j}(t)\right) \sin \tilde{\varphi}_{j}(t), \\
G_{2}(t)=\sum_{j=1}^{N} g_{j}(t)\left(-\frac{K_{\mathrm{t}}}{K_{\mathrm{r}}} \cos \left(2 \tilde{\varphi}_{j}(t)\right)-\sin \left(2 \tilde{\varphi}_{j}(t)\right)\right) \\
\times \sin \tilde{\varphi}_{j}(t) .
\end{array}
$$

Equation (21) is a linear delay-differential equation, which includes a periodically varying stiffness (associated with $\left.G_{1}(t)\right)$ and a periodically varying damping (associated with $G_{2}(t)$ ). The time-periodic damping is a new term compared to standard milling models [21-26]. By omitting this term, Eq. (21) reduces to the governing equation of milling models without velocity-dependent projections of the chip thickness and the cutting force. The time-periodic damping is inversely proportional to the nominal cutting speed $R \Omega$. If $G_{2}(t)$ were a positive constant, then this term would provide an additional damping for low spindle speeds, which is known as the process damping effect $[3-6,10,14]$. Note that $G_{2}(t)$ is actually time periodic and, as will be shown in Sect. 5 , may become negative.

In Sect. 5, we investigate the time-periodic coefficient $G_{2}(t)$ and its effects on the stability lobe diagrams of milling. We compare the results to those derived for turning operations. Substituting $G_{1}(t) \equiv 1$ and $G_{2}(t) \equiv K_{\mathrm{t}} /\left(q K_{\mathrm{r}}\right)$ into Eq. (21) gives the case of turning processes, for which the process damping effect was analyzed in $[4,14]$ with $q=1$. The case $G_{1}(t) \equiv 1$, $G_{2}(t) \equiv K_{\mathrm{t}} /\left(q K_{\mathrm{r}}\right)$ occurs for $N=1, \tilde{\varphi}_{j}(t) \equiv \pi / 2$, and $g_{j}(t) \equiv 1$. That is, the special case of turning can be investigated by considering a single tooth permanently in cut at $90^{\circ}$ angular position.

In order to reduce the number of parameters, let us write Eq. (21) in dimensionless form by introducing the dimensionless time $\hat{t}=\omega_{\mathrm{n}} t$, the dimensionless angular velocity $\hat{\Omega}=\Omega / \omega_{\mathrm{n}}$, and the dimensionless delay $\hat{\tau}=$ 
$\omega_{\mathrm{n}} \tau=2 \pi /(N \hat{\Omega})$. The derivative with respect to $\hat{t}$ is indicated by prime and satisfies $\dot{\xi}(t)=\omega_{\mathrm{n}} \xi^{\prime}(\hat{t})$. After dropping the hat, we get

$$
\begin{aligned}
\xi^{\prime \prime}(t) & +2 \zeta \xi^{\prime}(t)+\xi(t) \\
= & -p G_{1}(t)(\xi(t)-\xi(t-\tau))-p \tau \rho G_{2}(t) \xi^{\prime}(t)
\end{aligned}
$$

where $p=H / \omega_{\mathrm{n}}^{2}$ is the dimensionless axial depth of cut, and $\rho=f_{z} N /(2 R \pi)$ is the dimensionless feed per tooth. Note that substituting $\rho=0$ reduces Eq. (24) to the governing equation of milling models without process damping, whereas the substitution $G_{1}(t) \equiv 1$, $\rho G_{2}(t) \equiv C$ yields Eq. (1) of turning.

\section{Process Damping Coefficient and Stability Charts}

The stability of Eq. (24) was analyzed using the semidiscretization method (for more details, see [20]). Here we restrict ourselves to linear stability analysis. Analyzing the global behavior of the system is out of scope of this paper. For bifurcation and global stability analysis of milling processes, the reader is referred to [27]. Note that apart from semi-discretization, there exist several other approaches for computing the linear stability charts of milling, see e.g. the multi frequency solution [28, 29], the Chebyshev collocation [30, 31], the homotopy perturbation method [32], or the multi-mode approach [33].

Figure 4 presents the stability lobe diagrams for milling with a four-fluted tool $(N=4)$, damping ratio $\zeta=0.02$, cutting-force ratio $K_{\mathrm{r}} / K_{\mathrm{t}}=0.3$, and cuttingforce exponent $q=3 / 4$. During semi-discretization, the regenerative delay was resolved by 150 intervals and the charts were computed on a $600 \times 300$ grid in the plane $(\Omega, p)$. The stability lobe diagrams are presented for nine different radial immersion ratios: up-milling with $a_{\mathrm{e}} / D=0.02,0.2,0.6$, and 0.8 , full-immersion milling, and down-milling with $a_{\mathrm{e}} / D=0.7,0.5,0.1$, and 0.02 . For each case, two stability charts were computed with $\rho=0$ and $\rho=0.01$. When $\rho=0$, the time-periodic damping in Eq. (24) vanishes and we get the stability lobe diagrams of standard milling models without considering velocity-dependent chip thickness expressions and cutting force projections. In this case, the lower envelope of the stability lobes is a horizontal straight line, it is independent of the angular velocity $\Omega$. When $\rho=0.01$, we can observe the effect of the additional time-periodic damping (the process damping) on the stability charts. The periodic coefficients $G_{1}(t)$ and $G_{2}(t)$ corresponding to the various radial immersion ratios are also presented with blue and red lines, respectively.
It can be observed that modeling the velocity-dependent cutting force introduces a strong low-speed stability improvement in the mechanical model of milling operations with large radial immersion, see the up-milling with $a_{\mathrm{e}} / D=0.8$, the full immersion milling, and the down-milling with $a_{\mathrm{e}} / D=0.7$. In these cases, the timeperiodic damping coefficient $G_{2}(t)$ is mostly positive. Accordingly, the stability lobes for $\rho=0.01$ shift upwards at low cutting speeds compared to the case $\rho=0$, and there is a small improvement in stability at large cutting speeds as well. Thus, the velocity-dependent cutting force model captures the low-speed stability improvement for large radial immersion ratios.

However, as the radial immersion ratio is decreased (see the up-milling with $a_{\mathrm{e}} / D=0.6$ and the downmilling with $a_{\mathrm{e}} / D=0.5$ ), the low-speed stability improvement becomes less pronounced. The time-periodic coefficient $G_{2}(t)$ becomes negative for a larger portion of the period and the growth of the stable region is smaller. Decreasing the radial immersion ratio further, the low-speed stability improvement vanishes, see the cases of up-milling with $a_{\mathrm{e}} / D=0.2$ and down-milling with $a_{\mathrm{e}} / D=0.1$. In these cases, the time-periodic damping coefficient $G_{2}(t)$ is almost never positive. Correspondingly, the stability lobes for $\rho=0.01$ start to shift downwards at low cutting speeds compared to the standard models with $\rho=0$. Finally, for very low radial immersions (up-milling with $a_{\mathrm{e}} / D=0.02$ and downmilling with $a_{\mathrm{e}} / D=0.02$ ), the coefficient $G_{2}(t)$ is never positive, and the stability lobes shift towards zero depth of cut at low cutting speeds when $\rho=0.01$. This indicates the presence of negative process damping at low radial immersions.

The negative process damping contradicts experimental observations [3-10] that the stable region is larger at low cutting speeds. Therefore, the most important conclusion of this paper is that this kind of velocity-dependent cutting force model is not always able to capture the phenomenon of low-speed stability improvement. Although the model results in a positive process damping and improved low-speed stability for turning processes and milling with large radial immersion, it gives a negative process damping with a decrease in stability for low radial immersion milling.

\section{Geometric Illustration of the Process Damping Force}

In this section, we give a geometric illustration to explain why the model results in a negative process damping for milling with low radial immersion. We construct the additional process damping force originating in the velocity-dependent chip thickness expression and the 


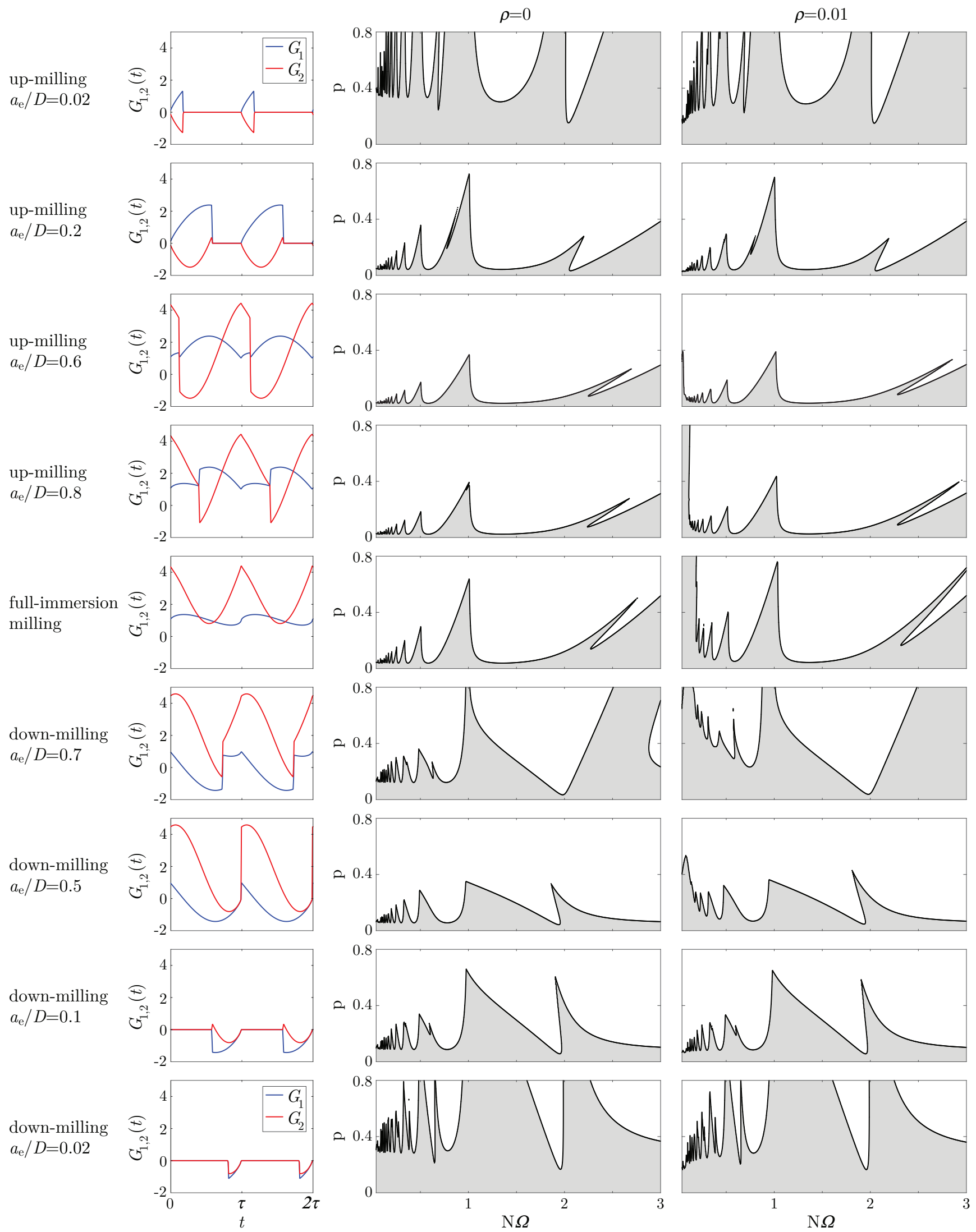

Fig. 4 Time-periodic coefficients of the governing equation of milling (blue: stiffness, $G_{1}(t)$, red: damping, $\left.G_{2}(t)\right)$ and the corresponding stability lobe diagrams $(\rho=0$ : standard milling model, $\rho=0.01$ : velocity-dependent model with process damping) 
cutting force projections. We demonstrate the results for a single tooth of the mill, hence we drop the subscript $j$. We consider those time instants where the tooth is engaged in cutting $(g(t) \equiv 1)$, and assume a linear cutting force characteristics $(q=1)$. For simplicity, we also omit the argument $t$.

Let $\tilde{\mathbf{F}}$ and $\mathbf{F}$ denote the nominal cutting force calculated with the standard chip thickness expression $\tilde{h}$ and the actual one obtained from the velocity-dependent model with chip thickness $h$, respectively. Let their difference be denoted by $\Delta \mathbf{F}=\mathbf{F}-\tilde{\mathbf{F}}$. We introduce chip thickness vectors with a similar notation: $\tilde{\mathbf{h}}=\tilde{h} \tilde{\mathbf{r}}$, $\mathbf{h}=h \mathbf{r}$, and $\Delta \mathbf{h}=\mathbf{h}-\tilde{\mathbf{h}}$, see Fig. 5 for rake angle $\alpha_{\mathrm{r}}$. Note that the rake angle $\alpha_{\mathrm{r}}$ indicated in the figure was chosen only for illustration purposes. The cutting force expression itself is independent of the rake angle, since only the direction of the actual cutting velocity is taken into account when calculating the cutting force. Similarly, changes in the tool's rake and flank face due to tool wear do not modify the cutting force expression of this model. The tool geometry may affect only the cutting-force coefficients $K_{\mathrm{t}}$ and $K_{\mathrm{r}}$.

According to Eq. (13), assuming a single tooth in cut with linear cutting force characteristics, the cuttingforce components become

$\tilde{F}_{\tilde{\mathrm{r}}}=K_{\mathrm{r}} a_{\mathrm{p}} \tilde{h}, \quad \tilde{F}_{\tilde{\mathrm{t}}}=K_{\mathrm{t}} a_{\mathrm{p}} \tilde{h}$,

$F_{\mathrm{r}}=K_{\mathrm{r}} a_{\mathrm{p}} h, \quad F_{\mathrm{t}}=K_{\mathrm{t}} a_{\mathrm{p}} h$.

Since $\tilde{F}_{\tilde{\mathbf{r}}} / \tilde{F}_{\tilde{\mathrm{t}}}=F_{\mathrm{r}} / F_{\mathrm{t}}=K_{\mathrm{r}} / K_{\mathrm{t}}$, the angle between $\tilde{\mathbf{r}}$ and $\tilde{\mathbf{F}}$ and between $\mathbf{r}$ and $\mathbf{F}$ are both equal to $\gamma=$ $\arctan \left(K_{\mathrm{t}} / K_{\mathrm{r}}\right)$. Besides, since $F_{\mathrm{r}} / \tilde{F}_{\tilde{\mathrm{r}}}=F_{\mathrm{t}} / \tilde{F}_{\tilde{\mathrm{t}}}=h / \tilde{h}$, the vector triangles $(\mathbf{h}, \tilde{\mathbf{h}}, \Delta \mathbf{h})$ and $(\mathbf{F}, \tilde{\mathbf{F}}, \Delta \mathbf{F})$ are similar. Consequently, the additional force $\Delta \mathbf{F}$ can be plotted by rotating $\Delta \mathbf{h}$ clockwise with angle $\gamma$.

The additional force $\Delta \mathbf{F}$ is responsible for the process damping effect in our model. If the force points to the right, then its $x$-directional component $\Delta F_{x}$ is positive and a positive process damping is introduced in the system. If $\Delta \mathbf{F}$ points to the left, a negative process damping term appears in the governing equation.

According to Fig. 5, the process damping force $\Delta \mathbf{F}$ forms an angle $\gamma-(\pi-2 \tilde{\varphi})-\alpha$ with the $y$ axis, where $\alpha=\tilde{\varphi}-\varphi$ is the angle between the chip thickness vectors $\tilde{\mathbf{h}}$ and $\mathbf{h}$ or, equivalently, between the nominal and the actual cutting velocities $\tilde{\mathbf{v}}$ and $\mathbf{v}$. Considering small vibration velocities (small $\dot{x}$ ), the angle $\alpha$ is also small and can be neglected compared to $\gamma$. Therefore, the angle of $\Delta \mathbf{F}$ can be approximated by $\gamma-(\pi-2 \tilde{\varphi})$. When the process damping is positive, the force $\Delta \mathbf{F}$ points to the right and the inequality

$0<\gamma-(\pi-2 \tilde{\varphi})<\pi$

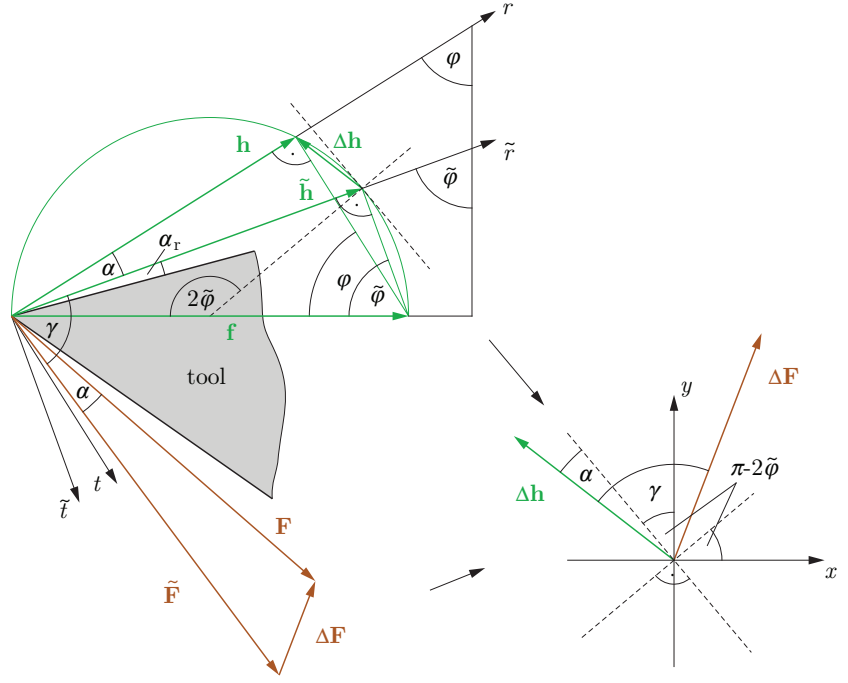

Fig. 5 Direction of the process damping force

holds. Thus, the process damping is positive if

$\varphi_{\text {ex }, \mathrm{cr}}<\tilde{\varphi}<\varphi_{\mathrm{en}, \mathrm{cr}}$.

where $\varphi_{\text {ex }, \mathrm{cr}}=\pi / 2-\gamma / 2$ and $\varphi_{\mathrm{en}, \mathrm{cr}}=\pi-\gamma / 2$ are the critical exit and enter immersion angles.

For low radial immersions, when $\varphi_{\mathrm{ex}}<\varphi_{\mathrm{ex}, \mathrm{cr}}$ in upmilling or when $\varphi_{\mathrm{en}}>\varphi_{\mathrm{en}, \mathrm{cr}}$ in down-milling, inequality (27) does not hold when the tooth is cutting. Therefore, the process damping is always negative. Note that this additional negative damping does not necessarily mean instability for systems with periodic coefficients (parametric excitation), but, as rule of thumb, one can say that the stable region is usually smaller for negative periodic damping coefficients than for positive ones.

In the example of Sect. $5, K_{\mathrm{r}} / K_{\mathrm{t}}=0.3$ and $\gamma=$ $\arctan \left(K_{\mathrm{t}} / K_{\mathrm{r}}\right)=73^{\circ}$, which yields $\varphi_{\mathrm{ex}, \mathrm{cr}}=53^{\circ}$ and $\varphi_{\text {en }, \mathrm{cr}}=143^{\circ}$. The direction of the process damping force in this example is illustrated for five different angular positions of the tooth assuming zero rake angle in Fig. 6. In the figure, the vibration velocity $\dot{x}$ and the angle $\alpha$ are enlarged for better visibility, they are no longer small, which slightly modifies the critical enter and exit immersion angles $\varphi_{\text {ex,cr }}$ and $\varphi_{\text {en,cr }}$. When $\tilde{\varphi}=$ $45^{\circ}<\varphi_{\text {ex }, \text { cr }}$ (see Fig. 6a) and when $\tilde{\varphi}=160^{\circ}>\varphi_{\text {en }, \mathrm{cr}}$ (see Fig. 6e), the force $\Delta \mathbf{F}$ points to the left implying a negative process damping. For $\tilde{\varphi}=\varphi_{\text {ex }, \text { cr }}$ (see Fig. 6b) and $\tilde{\varphi}=\varphi_{\text {en,cr }}$ (see Fig. $6 \mathrm{~d}$ ), the force $\Delta \mathbf{F}$ is vertical, hence the process damping term vanishes. When $\varphi_{\text {ex }, \mathrm{cr}}<\tilde{\varphi}=90^{\circ}<\varphi_{\text {en,cr }}$ (see Fig. 6c), the force $\Delta \mathbf{F}$ points to the right indicating a positive process damping.

Recall that the special case of turning operations is obtained by considering a single tooth permanently in 

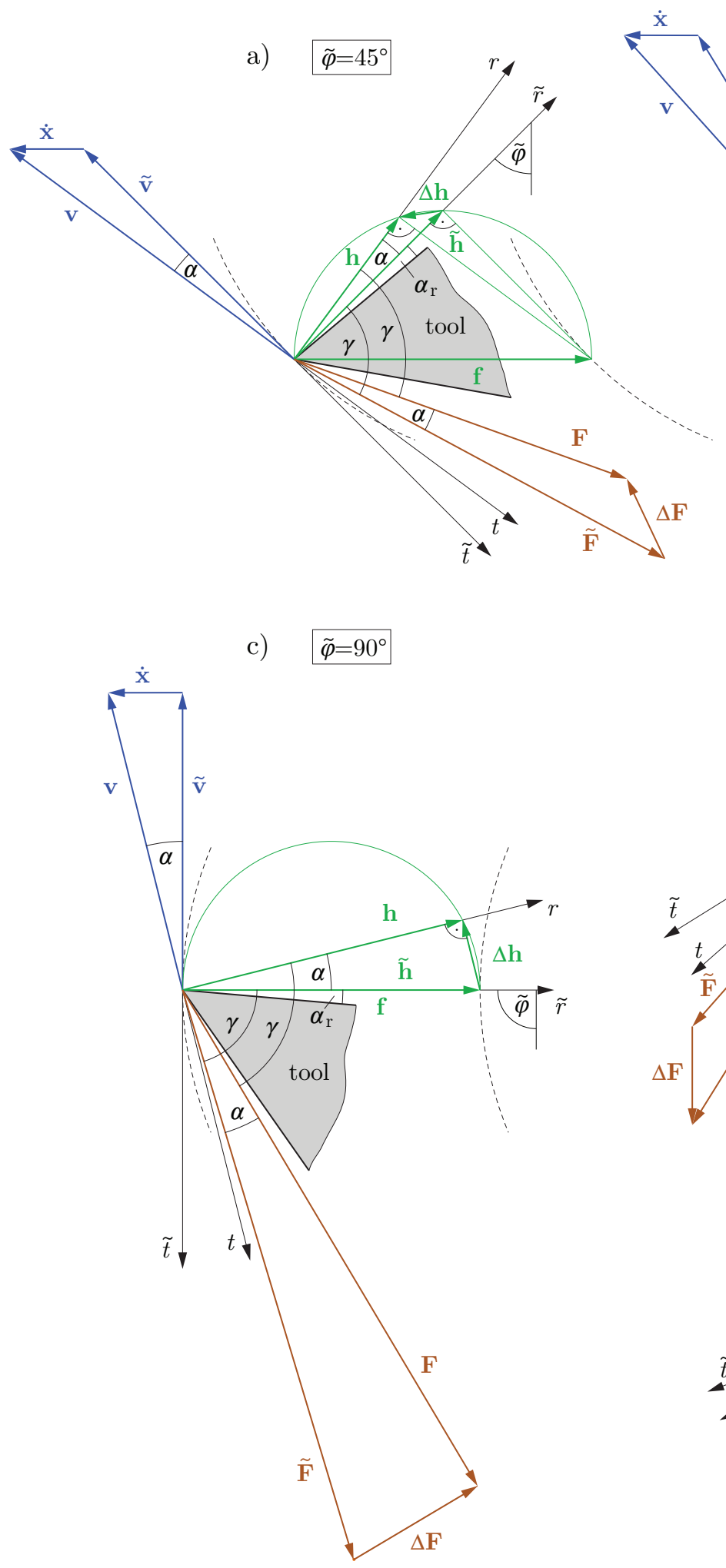
b) $\widetilde{\varphi}=\varphi_{\mathrm{ex}, \mathrm{cr}}$

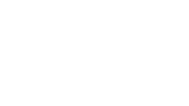

d) $\tilde{\varphi}=\varphi_{\mathrm{en}, \mathrm{cr}}$
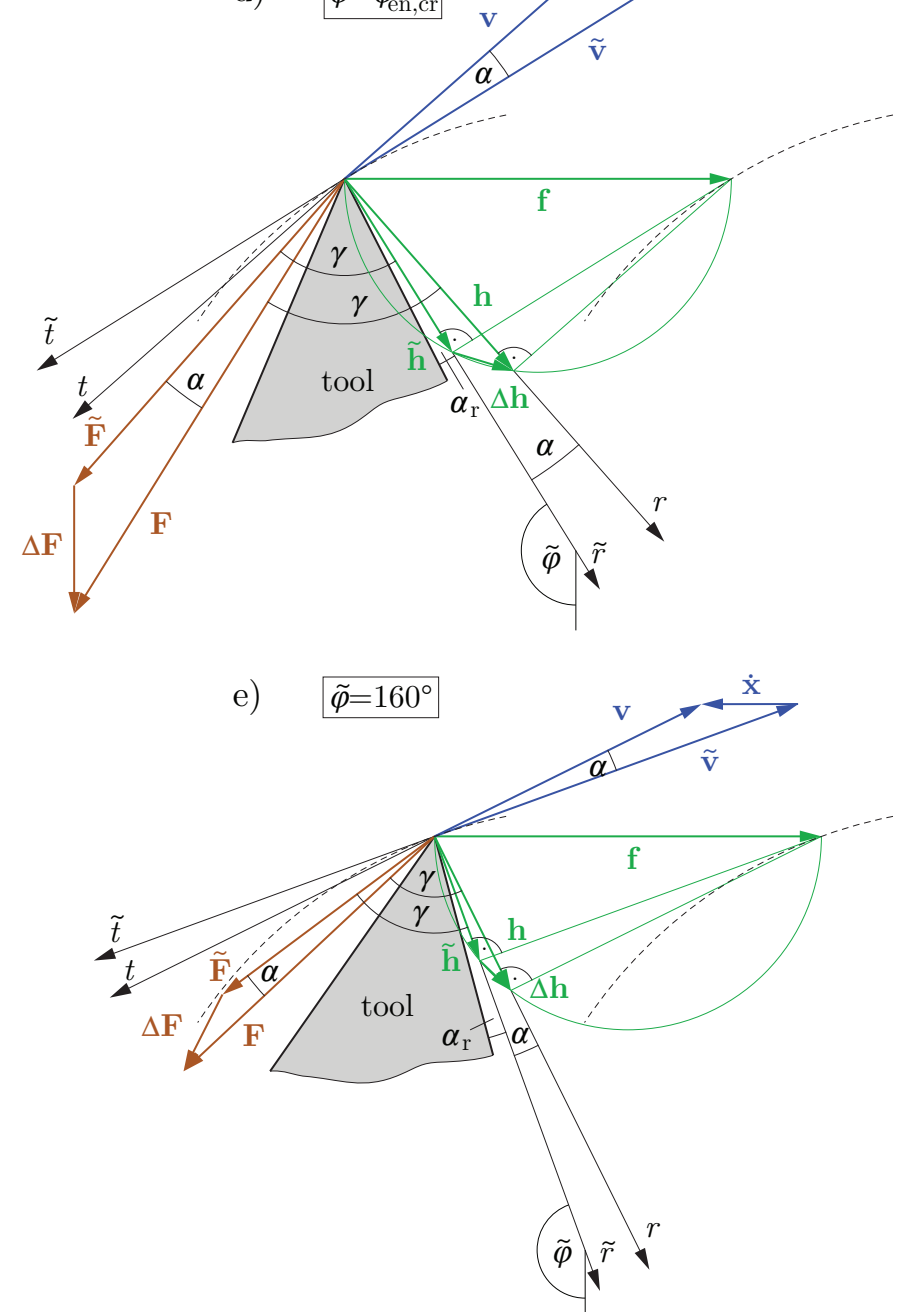

Fig. 6 Direction of the process damping force $\Delta \mathbf{F}$ for various angular positions of the cutting tooth 
cut at position $\tilde{\varphi}=90^{\circ}$. Based on Fig. $6 \mathrm{c}$, the velocitydependent cutting force model indeed introduces a strong positive process damping for turning operations. In this case, the vibration velocity $\dot{x}$ is perpendicular to the nominal cutting velocity $\tilde{\mathbf{v}}$. However, for low radial immersion milling, when $\tilde{\varphi}$ is close to $0^{\circ}$ or to $180^{\circ}$, and the vibration velocity $\dot{x}$ is almost parallel to the nominal cutting velocity $\tilde{\mathbf{v}}$, one gets a negative process damping.

Finally, a critical immersion ratio can also be determined, below which always a negative process damping occurs. According to Eqs. (15) and (27), the process damping coefficient is never positive for up-milling with radial immersion ratio $a_{\mathrm{e}} / D<\left(1-\cos \varphi_{\mathrm{ex}, \mathrm{cr}}\right) / 2$ and for down-milling with $a_{\mathrm{e}} / D<\left(1+\cos \varphi_{\mathrm{en}, \mathrm{cr}}\right) / 2$. In the above example, the critical cases correspond to $a_{\mathrm{e}} / D \approx$ 0.2 up-milling and $a_{\mathrm{e}} / D \approx 0.1$ down-milling. In Fig. 4 , the stability lobe diagrams are presented for these radial immersion ratios for a four-fluted tool $(N=4)$ with nonlinear cutting force expression $(q=3 / 4)$. We can see that indeed the process damping and the lowspeed stability improvement vanish approximately at these radial immersion ratios.

\section{Conclusions}

In this paper, we investigated the process damping effect for the single-degree-of-freedom model of milling by considering a velocity-dependent cutting force model following $[4,14,18]$. We took into account that the cutting velocity is affected by the vibration velocity during chatter. The direction of the actual cutting velocity was used to calculate the chip thickness and to project the cutting force to tangential and radial directions. Using the resulting velocity-dependent cutting force expression, the linearized equation of motion involves an additional time-periodic damping term, which is inversely proportional to the cutting speed. This additional damping is a kind of process damping, which may be responsible for low-speed stability improvement.

Analyzing the linear stability of the governing timeperiodic delay-differential equation, we computed the stability lobe diagrams of the system and arrived at an important observation. The experimentally observed phenomenon [3-10] that the stability lobes shift towards higher depths of cut at low cutting speeds is often modeled by adding a positive constant process damping term to the governing equation, which is inversely proportional to the cutting speed. This term can be explained by a cutting force model with velocitydependent chip thickness expression and force projections in case of turning operations [4, 14]. This explanation is also valid for milling operations with large radial immersion. However, the velocity-dependency introduces a periodically varying negative process damping for milling with low radial immersion. In this case, there is a decrease in the maximum stable depth of cut at low cutting speeds, which contradicts widely accepted experimental observations. Namely, a significant amount of experimental data has been reported in the literature, which verifies the existence of the low-speed stability improvement, that is, the increase of the maximum stable depth of cut at decreasing spindle speeds $[3-10,14]$. However, the authors are not aware of publications where the decrease of the low-speed stability was shown by experiments. Consequently, the velocitydependent cutting force does not provide a proper explanation to the low-speed stability improvement for all machining operations, and an extended model is needed for low radial immersion milling.

The improved stability at low cutting speeds can be attributed to other physical phenomena. One candidate is the interference between the tool's flank face and the wavy surface of the workpiece $[3-6,8-12,15]$. The flank contact force is modeled as a damping inversely proportional to the cutting speed as a first approximation, which gives a similar constant process damping term in the governing equation. Note, however, that the contact between the tool's flank and the workpiece is intermittent, it depends on the vibrations of the tool-workpiece system, which in fact yields complicated nonsmooth dynamics [12]. Stability properties of such nonsmooth systems can hardly be captured by linear models such as the one with constant process damping term.

Another possible explanation of the low-speed stability improvement is the so-called short regenerative effect $[16,17]$. These models account for the fact that the cutting force is the resultant of a force system distributed along the tool's rake face $[34,35]$. The governing equation of motion is a delay-differential equation where a short distributed delay is superimposed on the large regenerative point delay. The additional short delay originates in the fact that the chip needs a small amount of time to slip along the rake face of the tool. The additional short delay increases the maximum stable depth of cut at low cutting speeds for turning models $[16,17]$ and also for milling with any radial immersion [36]. Therefore, the short regenerative effect provides an appropriate model to explain the improved low-speed stability properties observed by experiments.

\section{References}

1. Tobias SA, Fishwick W (1958) Theory of regenerative machine tool chatter. The Engineer pp 199203, 238-239 
2. Tlusty J, Polacek M (1963) The stability of the machine tool against self-excited vibration in machining. In: ASME Production Engineering Research Conference, Pittsburgh, pp 454-465

3. Clancy BE, Shin YC (2002) A comprehensive chatter prediction model for face turning operation including tool wear effect. Int $\mathrm{J}$ Mach Tool Manu 42(9):1035-1044

4. Altintas Y, Eynian M, Onozuka H (2008) Identification of dynamic cutting force coefficients and chatter stability with process damping. CIRP Ann - Manuf Techn 57(1):371-374

5. Ahmadi K, Ismail F (2010) Experimental investigation of process damping nonlinearity in machining chatter. Int J Mach Tool Manu 50(11):1006-1014

6. Budak E, Tunc LT (2010) Identification and modeling of process damping in turning and milling using a new approach. CIRP Ann - Manuf Techn 59(1):403-408

7. Eynian M, Altintas Y (2010) Analytical chatter stability of milling with rotating cutter dynamics at process damping speeds. J Manuf Sci E-T ASME 132(2):021,012 (14 pages)

8. Ahmadi K, Ismail F (2012) Stability lobes in milling including process damping and utilizing multi-frequency and semi-discretization methods. Int J Mach Tool Manu 54-55:46-54

9. Shi Y, Mahr F, von Wagner U, Uhlmann E (2012) Chatter frequencies of micromilling processes: Influencing factors and online detection via piezoactuators. Int J Mach Tool Manu 56(2012):10-16

10. Jin X (2014) Identification of process damping coefficient based on material constitutive property. In: Proc of the ASME International Manufacturing Science and Engineering Conference, Detroit, MI, MSEC2014-4204

11. Stone E, Ahmed S, Askari A, Tat H (2005) Investigations of process damping forces in metal cutting. J Comput Methods Sci Eng pp 1-27, URL http://arxiv.org/abs/cs/0508102

12. Bachrathy D, Stépán G (2010) Time-periodic velocity-dependent process damping in milling processes. In: Altintas Y, Denkena B, Brecher C (eds) Proc of the 2nd International CIRP Conference on Process Machine Interaction, Vancouver, Canada, pp $1-12$

13. Quintana G, Ciurana J (2011) Chatter in machining processes: A review. Int $\mathrm{J}$ Mach Tool Manu $51(5): 363-376$

14. Altintas Y (2012) Manufacturing Automation Metal Cutting Mechanics, Machine Tool Vibrations and CNC Design, Second Edition. Cambridge University Press, Cambridge
15. Wan M, Ma YC, Feng J, Zhang WH (2016) Study of static and dynamic ploughing mechanisms by establishing generalized model with static milling forces. Int J Mech Sci 114:120-131

16. Stépán G (1997) Delay-differential equation models for machine tool chatter. In: Moon FC (ed) Dynamics and Chaos in Manufacturing Processes, John Wiley and Sons, New York, pp 165-192

17. Dombóvári Z, Stépán G (2010) Experimental and theoretical study of distributed delay in machining. In: Proc of the 9th IFAC Workshop on Time Delay Systems, Prague, Czech Republic, pp 1-5

18. Bachrathy D, Stépán G (2009) Bistable parameter region caused by velocity dependent chip thickness in milling process. In: 12th CIRP Conference on Modelling of Machining Operations, San Sebastian, Spain, pp 867-871

19. Tóth M (2014) Vibration analysis of milling processes. Master's thesis, Budapest University of Technology and Economics (Hungary)

20. Insperger T, Stépán G (2011) Semi-Discretization for Time-Delay Systems - Stability and Engineering Applications. Springer, New York

21. Altintas Y, Budak E (1995) Analytical prediction of stability lobes in milling. CIRP Ann - Manuf Techn 44(1):357-362

22. Insperger T, Mann BP, Stépán G, Bayly PV (2003) Stability of up-milling and down-milling, part 1: Alternative analytical methods. Int J Mach Tool Manu 43(1):25-34

23. Faassen RPH, van de Wouw N, Oosterling JAJ, Nijmeijer H (2003) Prediction of regenerative chatter by modelling and analysis of high-speed milling. Int J Mach Tool Manu 43(14):1437-1446

24. Faassen RPH, van de Wouw N, Nijmeijer H, Oosterling JAJ (2007) An improved tool path model including periodic delay for chatter prediction in milling. J Comput Nonlin Dyn-T ASME 2(2):167179

25. Wan M, Wang YT, Zhang WH, Yang Y, Dang JW (2011) Prediction of chatter stability for multipledelay milling system under different cutting force models. Int J Mach Tool Manu 51(4):281-295

26. Yang Y, Zhang WH, Ma YC, Wan M (2016) Chatter prediction for the peripheral milling of thinwalled workpieces with curved surfaces. Int J Mach Tool Manu 109:36-48

27. Dombóvári Z, Stépán G (2015) On the bistable zone of milling processes. P Roy Soc A-Math Phy 373:20140,409

28. Merdol SD, Altintas Y (2004) Multi frequency solution of chatter stability for low immersion milling. J Manuf Sci Eng-T ASME 126(3):459-466 
29. Bachrathy D, Stépán G (2013) Improved prediction of stability lobes with extended multi frequency solution. CIRP Ann - Manuf Techn 62(1):411-414

30. Butcher EA, Ma H, Bueler E, Averina V, Szabó Z (2004) Stability of linear time-periodic delaydifferential equations via Chebyshev polynomials. Int J Numer Meth Eng 59(7):895-922

31. Totis G, Albertelli P, Sortino M, Monno M (2014) Efficient evaluation of process stability in milling with Spindle Speed Variation by using the Chebyshev Collocation Method. J Sound Vib 333(3):646668

32. Compeán F, Olvera D, Campa FJ, López de Lacalle LN, Elías-Zúñiga A, Rodríguez C (2012) Characterization and stability analysis of a multivariable milling tool by the enhanced multistage homotopy perturbation method. Int J Mach Tool Manu 57:2733

33. Urbikain G, Olvera D, López de Lacalle LN, ElíasZúñiga A (2015) Stability and vibrational behaviour in turning processes with low rotational speeds. Int J Adv Manuf Tech 80(5):871-885

34. Childs THC, Mahdi MI (1989) On the stress distribution between the chip and tool during metal turning. CIRP Ann - Manuf Techn 38(1):55-58

35. Buryta D, Sowerby R, Yellowley I (1994) Stress distributions on the rake face during orthogonal machining. Int J Mach Tool Manu 34(5):721-739

36. Molnár TG, Insperger T (2015) On the effect of distributed regenerative delay on the stability lobe diagrams of milling processes. Period Polytech Mech $59(3): 126-136$ 